\title{
Introduction to Global Virtual Teams Mini-track
}

\author{
Michael J. Hine \\ Sprott School of Business \\ Carleton University \\ mike.hine@carleton.ca
}

\author{
Derrick L. Cogburn \\ School of International Service \\ American University \\ dcogburn@american.edu
}

The Global Virtual Teams (GVT) mini-track has existed since HICSS-42 and continues to attract many high-quality papers that push the frontiers of knowledge in global virtual team practice and research. The minitrack was founded by Frederick Zarndt, a practitioner with decades of sr. management GVT experience. The current mini-track co-chairs, Mike Hine and Derrick Cogburn, became involved at HICSS-43 and HICSS-44 respectively. To date, the GVT mini-track has supported the publication of over 45 stimulating and cutting edge papers, each unique in their contribution to the overall GVT body of knowledge.

Initial GVT mini-track solicitations had a focus on culture and language which reflected Frederick's own experiences working in GVT environments. While that topic remains fundamental to today's GVT research, the area has expanded greatly and now papers on leadership, creativity, power distance, emotion, information sharing, social loafing, collaboration and personality, all in the context of GVT are published regularly. In addition, insights gained from other platforms such as massive multiplayer online games (MMOG) and virtual worlds are being used to add insight into the increasingly complex GVT organizational form.

The fundament GVT research challenge remains similar to what it was 10 years ago, ie. how do we increase the effectiveness and efficiency of operating in distributed virtual settings? As humans are inherently complex there is still much work to be done exploring how cognitive and affective individual differences play out in global virtual environments. In addition, collaboration tools are constantly emerging and evolving creating new and exciting lines of inquiry. Because of the rapidly changing technological landscape, much of the previous GVT research needs to be re-evaluated and re-validated in the context of the newly available tools.

As the GVT organizational form has become more dominant, the type of work being executed has evolved as well, with an increased focus on strategic complex tasks that have a high degree of ambiguity associated with them. This shift, along with the unique challenge of working virtually with individuals and sub-groups from different countries, with different cultures and different first languages continues to ensure that global virtual research is an exciting and relevant topic of inquiry.

The following five papers were accepted to this year's mini-track. In the first paper: The Perceived Level of Collaborative Environment's Effect on Creative Group Problem Solving in a Virtual and Distributed Team Environment, Bozan investigates team members' perceived level of collaboration on creative group problem solving in virtual and distributed IS project settings. An empirical model is tested and recommendations for enhancing creative problem solving in virtual and distributed IS project environments are presented. The second paper: Towards Genuine Virtual Collaboration: Designing the Use of Mobile Remote Presence (MRP) in Offshore-Outsourced Projects by Wende, Alt and King reports on the usage of an MRP system by a distributed team over a period of a year. The paper also provides a plan for a rollout of an MRP system in an offshore outsourced project with team members in India and in Germany. The third paper: Cultural Influences on Collaborative Work in Software Engineering Teams by Fazli and Bittner provide a comprehensive literature review on cross-cultural software engineering. The paper details potential impacts of national cultural factors on collaborative approaches and behavior in software engineering teams. The fourth paper: Shared Mental Models in Creative Virtual Teamwork by Redlich, Siemon, Lattemann, and Robra-Bissantz reports on the usage of an online whiteboard and its influence on the creation of Shared Mental Models (SMM) in early stages of virtual work. They find that specific 'creative competencies' of the technology can improve SMM but the perceived efficiency of the technological intervention lacks in comparison to being physically collocated with team members. Finally, the fifth paper: Building Accessible Cyberinfrastructure in the Global Disability Community: Evaluating Collaboration Readiness and Use of the DID Policy Collaboratory by Cogburn, Trevisan, Spaniol and Aguilar use survey results and observational data to gain 
insight into the socio-technical infrastructure required to enhance participation of the global disability community in key global governance processes.

We are extremely excited about the inclusion of the GVT mini-track in HICSS-50. It is a privilege to bring you these exciting papers and we look forward to having productive and stimulating discussions about current and future GVT issues. 